\title{
Dynamic Tension Between Success in a Surgical Career and Personal Wellness: How Can We Succeed in a Stressful Environment and a "Culture of Bravado"?
}

\author{
Charles M. Balch, MD FACS ${ }^{1}$ and Tait S. Shanafelt, MD $^{2}$ \\ ${ }^{1}$ Johns Hopkins Medical Institutions, Baltimore, MD; ${ }^{2}$ Mayo Clinic, Rochester, MN
}

\begin{abstract}
"Many cancer surgeons are troubled by commonly occurring stressors, which may be masked by the bravado of the surgical culture...It is time to recognize and proactively address the problems of burnout and poor quality of life in physicians.... Our data show that burnout, and its associated problems, are truly widespread occupational hazards that deserve a systematic, organizational intervention... Tension exists between a culture of productivity and the need for self-care". ${ }^{1}$

"Getting to wellness in all its dimensions and maintaining wellness throughout a career requires a mutual pact between the individual surgeon and the leadership that control their workplace environment.... Ultimately, quality outcomes for our patients may be enhanced if greater wellness can be cultivated in our surgical teams".
\end{abstract}

In this issue of the Annals are two valuable publications from the prestigious Memorial Sloan Kettering Cancer Center (MSKCC) in New York City. ${ }^{1,2}$ Guest and colleagues surveyed their surgical faculty using the standard Maslach Burnout Inventory and asked for surgeons' input regarding causes of distress and potential solutions. Although it represents a single-center experience with a relatively small sample size, the results were compelling: $\sim 40 \%$ were burned out, $27 \%$ had psychiatric levels of distress, and only $\sim 1 / 3$ reported high quality of life across physical, emotional, spiritual, and intellectual domains. Also, 30\% reported potentially problematic alcohol use, while $13 \%$ used sleep medications as a possible means to cope. $^{1,2}$ The MSKCC surgeons also frequently reported a

(C) Society of Surgical Oncology 2011

Published Online: 3 March 2011

C. M. Balch, MD FACS

e-mail: balchch@jhmi.edu putting personal life "on hold" to cope with stress, consistent with the well-described psychology of delayed gratification reported to be commonly used by physicians to deal with excessive work demands and stress. ${ }^{3,4}$

The MSKCC study, a collaborative effort between the Departments of Surgery, Psychiatry, and Employee Health, highlighted major causes of distress among their surgical faculty and solicited valuable information about modifiable solutions; their publications represent a refreshing transparency of reporting that should be a model for other institutions to follow.

\section{ARE THESE RESULTS REPRESENTATIVE?}

In 2008, the American College of Surgeons (ACS) Committee on Physician Health and Competency commissioned the team at Mayo Clinic to conduct a survey of its membership using the same validated survey instruments involving burnout, quality of life (QOL), and career satisfaction. ${ }^{5}$ This survey had an unprecedented sample size of 7905 surgeons from all surgical disciplines, age groups, and geographic settings. Collectively, $40 \%$ of surgeons met the criteria for burnout, $30 \%$ screened positive for depression, and $28 \%$ had a mental QOL score at least a $1 / 2$ standard deviation below that of the U.S. population..$^{5}$ In a multivariate analysis, the independent factors that were associated with burnout included: surgical subspecialty (odds ratio: $1.2-1.6 ; P<0.013$ ), having children between the ages of 5 and 21 years (OR: 1.35-1.41, $P<0.002)$, income entirely based on patient care billing (OR: 1.32; $P<0.001$ ), hours worked per week (OR 1.02 for each additional hour; $P<0.001$ ), and number of nights on call per week (OR: 1.06 for each additional night; $P<0.001)$. Younger surgeons and those with children between the ages of 5 and 21 were at higher risk as were surgeons whose compensation was based entirely on billing/productivity, and those who spent more nights on call 
per week. The absence of burnout and subspecialty choice was among the most important factors associated with career satisfaction. ${ }^{5}$

Skeptics may have difficulty believing these national survey data indicating a $40 \%$ burnout rate apply to the surgical faculty at their own institution. The results from MSKCC and a similar unpublished survey of 77 Johns Hopkins surgical faculty and trainees yielded nearly identical burnout rates (J. Freischlag, personal communication) to the ACS study. Two national studies of surgical oncologists specifically showed similar findings. ${ }^{6,7}$ Compared with surgeons from all other specialties, surgical oncologists had a similar incidence of burnout, suicide ideation (SI), and mental/physical QOL, but a lower incidence of depression, and better indices of career satisfaction. Despite these generally favorable findings, surgical oncologists reported greater challenge regarding having a schedule that leaves enough time for personal and family life and experiencing a higher prevalence of work-home conflicts compared with other surgical specialists. Thus, the MSKCC results about the incidence of surgeon distress appear representative of surgeons in similar practice settings, even though the specific contributors and solutions for addressing them may vary somewhat from center to center.

\section{ASSOCIATIONS BETWEEN BURNOUT AND BOTH MEDICAL ERRORS AND SI}

Increasing evidence suggests that physician burnout can adversely affect patient safety, quality of patient care, and even contribute to medical errors. ${ }^{8,9}$ In the ACS study, major medical errors reported by surgeons are strongly related to a surgeon's degree of burnout and their mental QOL. For example, reporting a major medical error in the last 3 months was associated with a 7-point increase (59\% of the standard deviation, e.g., a large effect size) in emotional exhaustion on the MBI and roughly a doubling in the risk of screening positive for depression (27.5 vs. 54.9\%; $P<0.0001)$. Burnout and depression remained independent predictors of reporting a recent major medical error on multivariate analysis that controlled for other personal and professional factors. Although the cross-sectional nature of ACS survey cannot discern cause and effect relations, longitudinal studies in residents suggest burnout does indeed increase the risk of error. ${ }^{10,11}$

Collectively, the findings underscore that surgeons' mental health and professional burnout matter not only to the individual surgeon and their family, but to their patients, colleagues, societies, hospitals, and government agencies tasked with promoting quality of care. ${ }^{9,12,13}$ In addition to its potential impact on patient safety, physician burnout should also be of concern to healthcare organizations since workers who are less satisfied tend to be less productive and eventually may decide to quit for a different practice opportunity or take an early retirement. Medical errors and decreased patient satisfaction with the medical care provided by burned out physicians also increase the threat of malpractice litigation, and, hence, physician burnout also poses substantial risk to the economic wellbeing of healthcare organizations. In an accompanying editorial, Campbell stated, "What seems clear is that a broad strategy to reduce errors could logically be based on attention to both the individual and to the system in which the individual functions." and then stated, "It is logical to propose, based on the work by Shanafelt and other similar work, that hospitals interested in improving safety regularly survey the level of mental distress in their faculty as part of their error reduction strategy." 9

Surgeon burnout can also have potentially profound personal consequences for the individual surgeon, of which suicide is perhaps the most tragic example. In the ACS survey, SI was reported by 501 surgeons $(6.4 \%)$ during the previous 12 months. ${ }^{14}$ The prevalence of SI was $1.5-3.0$ times more common among surgeons relative to the general population and increased with the severity of burnout, independent of symptoms of depression. These factors are interconnected. In a multivariable logistic analysis, burnout, depression, and report of a recent medical error were strongly and independently associated with SI after controlling for other personal and professional characteristics. $^{14}$

\section{IMPORTANCE OF A SUPPORTIVE WORKPLACE ENVIRONMENT}

We have previously published recommendations for how to achieve meaning, balance, and satisfaction in a surgical oncology career and emphasized the importance of an efficient and supportive workplace environment. ${ }^{6,12,15}$ The MSKCC investigators also emphasize the importance of the workplace environment in what they appropriately characterize as a "mutual pact between the individual surgeon and the leadership that control their workplace environment." 1 They further state that leaders must acknowledge that "stressors have an adverse effect on faculty, and then take action to address modifiable factors that are most strongly associated with burnout... Hospital decision makers should realize that a surgeons' wellness appears to be greatly impacted by stress related to difficulties scheduling holidays, conferences, and time available to be with family." 1

Here, the MSKCC investigators offer a number of insights and solutions that were perceived to be important in their own workplace environment, some of which may be applicable to surgical oncology practices elsewhere. These recommendations include: $:^{1,2}$ 
- Creating a culture that promotes work-life balance and personal wellness

- Reducing the taboo against discussing personal distress that otherwise can become "a culture of bravado"

- Addressing problems with the reimbursement system, inadequate administrative support, and scheduling issues

- Establishing a mentoring program for faculty

\section{WHAT IS THE INDIVIDUAL SURGEON'S RESPONSIBILITY FOR WELLNESS AND SELF-CARE}

Each surgeon brings to the issue of personal wellness a complex array of risk factors and solutions related to their background, personality, family influences and responsibilities, surgical subspecialty, practice characteristics, and professional expectations (both internal and external). In an age of "personalized cancer therapies," isn't it logical to assume that when addressing physician wellness, the solutions should be personalized too? Every day, each practicing surgeon reading this article thinks about cancer prevention, diagnosis, and treatment. Shouldn't we be talking about prevention, diagnosis, and treatment of burnout as well?

The surgical oncologists in the MSKCC study perceived a need for change from a culture of bravado to one of ethos and self-care. ${ }^{1}$ Almost half of the participating surgeons reported tension between the time devoted to work versus time available to be with family. The stressors most strongly associated with QOL were: (1) the quality of relationship with spouse, (2) medical lawsuits, and (3) tension between time devoted to work versus family. ${ }^{1,2}$

In a classic article on midcareer physician burnout, Spickard and colleagues provide additional insights into causes and solutions for burnout: "There is ample evidence that physicians are caught in a web of pressures including financial deficits, Medicare/Medicaid audits, concern over fraud and abuse, and malpractice suits in which they perceive little control....Marital problems and stresses in the family... The best prevention for physician burnout is to promote personal and professional well-being on all levels: physical, emotional, psychological, and spiritual. This must occur throughout the professional life cycle of physicians, from medical school through retirement. It is a challenge not only for individual physicians in their own lives but also for the profession of medicine and the organizations in which physicians work." 16

Work/home conflicts appear to be a major contributor to surgeon burnout and are more common among women surgeons. Work/home conflicts accounted for a substantial contribution to burnout among both women and men in the ACS survey, especially among women surgeons. ${ }^{5,17}$
Although the factors contributing to burnout were remarkably similar among women and men surgeons, women surgeons were more likely to experience work/home conflicts than their male colleagues. Surgeons whose domestic partner is another physician also experience unique challenges balancing personal and professional life. ${ }^{17}$ Efforts to recruit and retain surgeons should include attention to the additional challenges faced by two physician couples. ${ }^{18}$

Having studied and written about these issues for some years, we would make the following recommendations to our surgical colleagues as individuals: (1) make a personal commitment to understand and promote your own work/ personal life balance; (2) periodically assess whether your are achieving such balance; (3) cultivate an awareness of the risk factors for burnout that apply to your professional and personal life and be able to assess where you fit on a "risk scale" (e.g., high, medium, or low risk); (4) take personal responsibility and make a commitment to practice good health habits, especially as it the context of your own practice setting; (5) periodically assess your state of wellness in all domains: physical, psychological, emotional, and spiritual; (6) establish a mentor/mentee relationship with one or more individuals; and (7) familiarize yourself with the types of physician assistance programs and related resources that are available at your institution or medical facility.

\section{SHOULDN'T WE HAVE MORE OF A NATIONAL DIALOGUE ON THIS ISSUE?}

An ever-increasing literature indicates that burnout, and its associated problems, are truly widespread occupational hazards for physicians that need to be addressed through strategic, systematic organizational initiatives. Surgical and hospital leaders should be able to identify the groups of physicians at greatest risk for burnout, but also recognize the unique and individual factors that contribute to the syndrome. One major benefit of studies such as those from MSKCC is that people are talking about the issue of physician burnout - at the organizational, hospital, departmental, and individual level. Proposed solutions are starting to appear in the literature. Just as evidence-based research drives our standards of care, so too should there be rigorous assessment of the efficacy of such solutions and practice reorganizations. Funding for such efforts is currently anemic and will be critical if progress is to be made.

Guest et al. ${ }^{1}$ conclude that: "Surgical leadership can begin by acknowledging that stressors have an adverse effect on faculty, and then take action to address modifiable factors that are most strongly associated with burnout and low quality of life." As a starting point, leadership efforts should incorporate mentoring programs, flexibility in 
scheduling, accountability, compassion, and appropriate administrative support and adapt solutions to the uniqueness of both their workplace environment and the diversity of their surgeons.

Each institution, and surgical specialty groups within it, is faced with a complex array of factors that contribute to burnout. Many solutions must be developed and applied at the local level. While not all of the issues identified by the MSKCC research team are applicable to all surgical groups and all settings, the process they followed to evaluate surgeon burnout at their center and to identify potential organizational opportunities to promote wellness is laudable and a reasonable model for other surgery departments to follow. We salute their commitment to explore this challenge and to share their unvarnished results through these publications, which reflect a thoughtful and compassionate concern by the MSKCC leadership for the wellbeing of their faculty, our profession, and ultimately for our patients.

\section{REFERENCES}

1. Guest R, Scardino P, et al. Cancer surgeons' distress and wellbeing, I: the tension between a culture of productivity and the need for self-care. Ann Surg Oncol. 2011.

2. Guest R, Scardino P, et al. Cancer surgeons' distress and wellbeing, II: modifiable factors and the potential for organizational interventions. Ann Surg Oncol. 2011.

3. Shanafelt T, Chung H, White H, Lyckholm LJ. Shaping your career to maximize personal satisfaction in the practice of oncology. J Clin Oncol. 2006;24:4020-6.

4. Gabbard GO, Menninger RW. The psychology of postponement in the medical marriage. JAMA. 1989;261:2378-81.

5. Shanafelt TD, Balch CM, Bechamps GJ, et al. Burnout and career satisfaction among American surgeons. Ann Surg. 2009;250: 463-71.
6. Kuerer HM, Breslin T, Shanafelt TD, Baile WF, Crawford W, Balch CM. Road map for maintaining career satisfaction and balance in surgical oncology. J Am Coll Surg. 2008;207:435-42.

7. Balch CM, Shanafelt TD, Sloan J, Satele DV, Kuerer HM. Burnout and career satisfaction among surgical oncologists compared with other surgical specialties. Ann Surg Oncol. 2011; 18:16-25.

8. Shanafelt TD, Balch CM, Bechamps G, Russell T, Dyrbye L, Satele D, et al. Burnout and medical errors among American surgeons. Ann Surg. 2010;251:995-1000.

9. Campbell DA, Jr. Physician wellness and patient safety. Ann Surg. 2010;251:1001-2.

10. West CP, Huschka MM, Novotny PJ, Sloan JA, Kolars JC, Habermann TM, et al. Association of perceived medical errors with resident distress and empathy: a prospective longitudinal study. JAMA. 2006;296:1071-8.

11. West CP, Tan AD, Habermann TM, Sloan JA, Shanafelt TD. Association of resident fatigue and distress with perceived medical errors. JAMA. 2009;302:1294-300.

12. Balch CM, Copeland E. Stress and burnout among surgical oncologists: a call for personal wellness and a supportive workplace environment. Ann Surg Oncol. 2007;14:3029-32.

13. Balch CM, Freischlag JA, Shanafelt TD. Stress and burnout among surgeons: understanding and managing the syndrome and avoiding the adverse consequences. Arch Surg. 2009;144:371-6.

14. Shanafelt TD, Balch CM, Dyrbye L, Bechamps G, Russell T, Satele D, et al. Special report: suicidal ideation among American surgeons. Arch Surg. 2011;146:54-62.

15. Shanafelt T. A career in surgical oncology: finding meaning, balance, and personal satisfaction. Ann Surg Oncol. 2008;15: 400-6.

16. Spickard A, Jr, Gabbe SG, Christensen JF. Mid-career burnout in generalist and specialist physicians. JAMA. 2002;288:1447-50.

17. Dyrbye LN, Shanafelt TD, Balch CM, et al. Relationships between work-home conflicts and burnout among American surgeons: a comparison by sex. Arch Surg. 2011;146:211-7.

18. Dyrbye LN, Shanafelt TD, Balch CM, et al. Physicians married or partnered to physicians: a comparative study in the American College of Surgeons. J Am Coll Surg. 2010;211:663-71. 